\section{CAN ANTITOXIN STATISTICS BE RELIED} UPON?

BY GUSTAVUS BLECH, A.B., M.D. SURgeon to THE GERMAN FREE DISPENSARY. DETROIT, MICH.

The many fiascoes in modern medicine have taught me a lesson, viz: Never to be the first and never to be the last one, to adopt a new remedy. Liberality is one of the first conditions for progress and success in medicine; conservatism, the preventative for poetic illusions and errors, undiscovered on account of the blinding effect of enthusiasm.

I have quietly watched the reports of cures of diphtheria with antitoxin, and have wondered how easy it is to catch the masses, even if one knows next to nothing about the modus operandi of a remedy. Beside, its origin and composition is mystery as yet, its preparation being controlled entirely by a few enterprising chemists. But neither this nor the fact that.its introduction into the human system is apt cause a good many dangers like leucocy themia, shall be brought as an argument against its value; even the fact, that a good many eminent and thoroughly reliable physicians have reported their experiments with it as failures shall not be mentioned, and only one modest question shall be asked: Can antitoxin statistics be relied upon? I dare answer no, not always. I do not doubt for one moment the honesty of the reporters and their noble intentions; for the sake of the honor of profession, I will say that "fig . ures will not lie," but I can not help assert that "figures will." The fact is, that every case reported as diphtheria, is indeed, not always true diphtheria. In some cases the microscopic examination alone was sufficient for a diagnosis, and that was false. In other cases the physical signs, the clinical picture was the medium, and that was false also. Both together, microscope and thermometer, eye and hand, must be employed to obtain a correct diagnosis.

Diphtheria, true diphtheria in spite of horse, jackass or any other quadruped's serum, is a grave, constitutional disease, the prognosis of which is always doubtful. It is exaggerated when one of my friends asserts, that he makes a diagnosis of true diphtheria post-mortem only; but there is some truth in that intended joke. There are plenty of poor children running around the streets with sore throats, follicular tonsillitis, rhinitis fibrinosa and never take a drop of medicine and get well. And in some of these a physician is called in, and a few hours later, the health inspector is placarding the house. If, by chance, the parents are smart enough to call in a more experienced physician, off goes the red sign. I had quite a number of such cases in my practice, but in one of my last ones, I had an encounter with our local board of health, which ended in my victory. The case I refer to is that of a little boy R. S., aged 4 years. He was taken sick with sore throat and nose, the latter bleeding on touch, membranes being expelled on sneezing. A reputable physician was called who pronounced it diphtheria. He sent a culture to the local board of health where Klebs-Loeffler bacilli were found. For some unknown reason to me, the parents discharged the physician and called me in to attend the case. I made a careful examination of the nose aud throat found a somewhat accelerated pulse and a temperature below 100 degrees $\mathrm{F}$. The boy ran from one room to another, and would not agree to be confined to bed. While the mucous membrane appeared to be, on the first look, diphtheria, a closer examination revealed the double malady of follicular tonsillitis and rhinitis fibrinosa. Both affections simulate diphtheria almost to delusion. The clinical picture of a grave constitutional disease, high fever, etc., which symptoms are a conditio sine qua non for a diagnosiz of true diphtheria, were absent. On the other hand some authors have lately classified rhinitis fibrinosa with diphtheria, but the best authorities in the old and the new world strongly object to such classification. The argument, that if the KlebsLoeffler bacillus be found, the case must necessarily be one of true diphtheria, does not always hold good, as Baginsky and others have found the same bacillus in different forms of rhinitis and pharyngitis. While I must admit, that not every case of diphtheria is associated with a high temperature, constitutional depression, etc., such is the rule without any excep. tion in nasal diphtheria. After nose and throat have been sprayed with hydrozone there could be found no microbe in the culture taken the other day.

The number of cases of this kind which I have successfully treated approaches closely to one hundred. If, according to the Board of Health of New York, Chicago and other institutions, they ought to be styled diphtheria, well then, what is the use of injecting blood serum taken from some animal? We have in hydrozone ( 30 volumes aqueous solution of anhydrous peroxid of hydrogen) a remedy which not only kills instantaneously the Loeffler bacilli, but also changes chemically the nature of the soil in which their spores can develop. Ite deadly action is limited to vegetable cells (pathogenic germs) and it is the most powerful stimulant to healthy granulations, having no injurious action upon healthy animal cells. In fact, my experiment taught me that hydrozone is a safe and most reliable remedy to use in the treatment of diphtheria. On the contrary, I will say; How can we rely upon antitoxin?

203 E. Columbia Street.

\section{A CASE OF STONE IN THE KIDNEY-NEPHRO- LITHOTOMY-RECOVERY.}

\section{BY W. D. HAMILTON, M.D.}

Since Dr. Morris, of Middlesex Hospital, elaborated the operation for the removal of a stone from the kidney, the procedure has been frequently and successfully employed. The diagnosis of that condition is often difficult. In a sweeping way it might be stated that the inconstancy of the symptoms has prevented precision in the recognition and location of the disease. Again, other pathologic renal affections have shown symptoms suggestive of stone in that organ. Repeated attacks of renal colic, blood and pus in the urine, with the occasional passage of sabulous matter per urethram are strongly suggestive of it. Jordan Lloyd's valuable test led the writer to the diagnosis of stone in the kidney in the case about to be nar. rated. The hypothesis of renal calculus was offered, however, with mental reservations; for one surgeon, Dr. Jacobson, of Guy's, has reported not less than twenty-five exploratory lumbar incisions, where the symptoms of stone having been present some other condition was found. Early tuberculosis of the kidney, malignant disease involving the renal substance, 
and the uric acid diathesis were the chief sources of error.

The inadequacy of puncturing the organ, and even its thorough palpation as final tests of the presence or absence of stone may be shown by the experience of Morris. The coterie of so-called stone symptoms was typical in a case in charge of this capable surgeon. Exploration through a lumbar cut, needling and renal incision brought negative results! The patient survived the operation. The symptoms recurred and we are compelled to admire the courage of Dr. Morris who removed the suspected kidney by the lumbar route. He could feel with the fingers no induration in its substance, when after nephrectomy the specimen lay before him. After incising it at several points he finally found, however, a stone of some size embedded in the renal tissue. The patient got well. Dr. Howard Kelley's recent article in the News will probably teach us much by the use of instruments of precision; for catherization of the ureters, perfected as he states, will show us at least the kind of work done by either kidney, The patient, Mr. A., æt. 49, a merchant from Newark, Ohio, was brought to the writer by Dr. Notring, of that place. He had been an invalid for three years. His appearance was that of a man accustomed to great suffering; he walked in such a way as to favor the left side; he was badly stooped, and the concavity of the bend of his body was to the front and left. His side having been exposed, he was given a sharp punch in the lame loin, the thumb being forced in the direction of the kidney. $\mathrm{He}$ complaimed that he felt as if a sword had been stuck into him. Jordan Lloyd looks upon a statement of this kind by the patient, after receiving such a thrust, as being strongly suggestive of the presence of renal calculus. A uranalysis at the time elicited the following facts: Sp. gr., 1012; reaction acid; phosphates present; no casts.

About forty attacks of renal colic had occurred within the three years of invalidism preceding his visit. Each meant a week's illness in bed. Pain began commonly in the night, starting in the left loin. A so-called swelling showed itself in that region during these seizures and disappeared after them. More or less complete anuria accompanied this intumescence, and was followed by a copious discharge of urine each time. Increasing intensity of this loin-ache with frequent and painful dribbling acts of urination, were invariable accompaniments of these paroxysms. The free administration of morphia was necessary during them. Neither pus nor blood nor sabulous matter in the urine was ever observed by the patient or the attending physician. To make things more obscure, in a violent lifting effort, made about the time that his illness began, a left inguinal hernia was produced. He naturally attributed much of his discomfort to this fact. $\mathrm{He}$ was told that he had a stone in the left kidney. An operation was performed at his home on Aug. 4., 1895. The usual preparation was employed. Strychnia was given beforehand in thirtieth grain doses for a few days, for its stimulant and tonic effect; while the parts were cleansed and suitably prepared to minimize the risk of sepsis; ether narcosis; right lateral position; a hard pillow was placed beneath the right loin, so that the left costo-iliac space was thus put on the stretch and increased. A four-inch oblique incision was made, avoiding the pleura above and peritoneum to the ventral side. The kidney was readily exposed; the finger was passed about the organ to separate the fatty capsule. On palpating its posterior surface toward the hilum a hard mass could be felt in the pelvis, which it well-nigh filled. Invaluable aid was rendered by the careful management of the anesthetic by Dr. Notring and by the strong upward traction of the ribs by Dr. Spear, so that the kidney could be brought well into the parietal opening. A probe was carried into the more accessible lower segment of the cortex, radially toward the pelvis, while the organ was steadied by the thumb and fingers. A stone was felt. The instrument in place served as a guide to the guarded bistoury with which the wound was enlarged; while a pair of angular Wells' forceps facilitated extraction of the stone. A drain was inserted into the loin; no vessels were ligated. Some shock supervened, but disappeared, however, within three hours. A few silk stitches were inserted. Some small sabulous fragments were discharged through the natural passages in the next few days. For a fortnight, urine was somewhat bloody, and afterward became cloudy.

Profiting by a previous experience in which after crushing a vesical calculus of the uric acid variety, the frequent voiding of cloudy urine had been a source of anxiety to the patient, the very free drinking of lithia water was advised and the urine became clear. He had quite recovered within a month so as to be able to pursue his occupation. The stone, a uric acid concretion, weighed $73.5 \mathrm{grs}$. It occupied the pelvis of the kidney which it almost filled, while the upper part of it lay embedded in the renal tissue. It might be well in closing to allude to the striking and misleading facts of the case, viz.: the absence of pus and blood and calcareous matter in the urine. $\mathrm{He}$ was at times, no doubt, a sufferer from acute hydro-nephrosis, as his history indicated.

\section{CLINICAL LECTURE.}

Extract from of a Clinical Lecture Delivered before the Senior Students at the Wisconsin College of Physicians and Surgeons.

BY JAMES A. BACH, M.D.

PROFESSOR OF OPHTHALMOLOGY AND OTOLOGY. MILWAUKEE, WIS.

Gentremen:-Our first patient to-day is one of unusual interest. This young lady, as she tells us, about three years ago had the misfortune to receive a slight injury of her right eye, which injury was followed by deep infection, producing a plastic iridocyclitis. In due course of time the fellow eye became affected with a sympathetic inflammation, which practically amounted to an extension of the infection through the optic nerve sheaths, leading to simjlar results, i.e., a plastic inflammation of the uveal tract with extensive adhesions. The inflammatory process in both eyes has now come to a standstill, with the result that the girl has simply a perception of light left.

Covering one eye and moving this light across different parts of the visual field you will notice that she readily locates the light correctly; in other words, her light projection is good, showing that the perceptive organs are, to say the least, partially intact in every portion of the field. The readiness with which she locates this light, although only a very small amount can pass into the interior of her eye, owing to these dense plastic exudates in the pupillary field, gives us a reasonable assurance that if we can suc- 